\title{
Photon acceleration versus frequency-domain interferometry for laser wakefield diagnostics
}

\author{
J. M. Dias, ${ }^{1}$ L. Oliveira e Silva, ${ }^{2}$ and J. T. Mendonça ${ }^{1}$ \\ ${ }^{1}$ GoLP/Centro de Física de Plasmas, Instituto Superior Técnico, 1096 Lisboa Codex, Portugal \\ ${ }^{2}$ Department of Physics and Astronomy, University of California Los Angeles, Los Angeles, California 90095
}

(Received 13 March 1998; published 6 July 1998)

\begin{abstract}
A detailed comparison between photon acceleration and frequency-domain interferometry, for laser wakefield diagnostics, is presented here. The dispersion effects on the probe beam and the implications of an arbitrary phase velocity of the plasma wave are discussed for both diagnostic techniques. In the presence of a large amplitude plasma wave and for long interaction distances, significant frequency up-shifts are observed. The importance of this effect on the determination of the phase and frequency shift measurements given by the two techniques is also analyzed. The accuracy of both diagnostics is discussed and some of their technical problems are reviewed. [S1098-4402(98)00004-4]
\end{abstract}

PACS numbers: 52.40.Nk, 52.35.Fp, 52.75.Di

\section{INTRODUCTION}

In recent years, there has been great interest in the generation of large amplitude electron plasma waves (EPW) because of their potential application for particle acceleration [1]. One of the most important goals of the research in the field of plasma particle accelerators is the development of experimental techniques to characterize the EPW generated by laser, for instance, in the laser wakefield accelerator or beatwave accelerator [1], or by an electron beam propagating through a plasma [2]. The first measurements of the temporal and spatial characteristics of the plasma waves generated by an ultrashort laser pulse (laser wakefield) were recently reported [3,4]. These experiments were based on the measurement of the phase shift experienced by a probe laser pulse using the frequency-domain interferometric technique (FDI) $[5,6]$.

In parallel, other experiments were performed with the purpose of studying the frequency up-shift resulting from the interaction of short laser pulses with relativistic ionization fronts $[7,8]$. An estimate for the velocity of the ionization front and its maximum electron density was obtained, clearly pointing to the feasibility of a new diagnostic tool, based on the measurement of the frequency shift experienced by a probe laser pulse interacting with coherent relativistic structures (e.g., laser wakefield) in laser produced plasmas [8].

In this paper, a detailed comparison of these two diagnostic techniques is carried out. A numerical simulation based on the ray-tracing equations for the probe laser pulse is employed to calculate the frequency shift due to photon acceleration of the laser pulse copropagating with the plasma wave. The same simulation also allows us to determine the phase shift of the laser pulse propagating in the laser wakefield relative to another laser pulse which propagates in the unperturbed plasma. Comparing both results we are then able to evaluate the importance of the frequency shift induced by the plasma wave on the determination of the phase shift. Comparatively, due to nonlinear [9] and 3D effects [10], it is clear that the group velocity of the driving beam responsible for the wakefield excitation can be considerably different from the velocity obtained from the linear dispersion relation in a plasma $\left(v_{g_{\text {linear }}}=c \sqrt{1-\omega_{p}^{2} / \omega^{2}}\right.$, where $\omega_{p}$ is the plasma frequency and $\omega$ is the laser pulse frequency). We have analyzed, for the first time, the regimes where the phase velocity of the wakefield can be considerably different from the group velocity of the probe pulse (which obeys the linear dispersion relation).

This paper is organized as follows. In Sect. II we present the basic principles of the two diagnostics, stressing the most significant technical characteristics of the FDI technique and pointing out the most important aspects of a photon acceleration diagnostic (PAD). In Sect. III we first present, for the sake of completeness, the ray-tracing equations used in the simulations and the calculation of the relative phase shift. The expressions for the wakefield scaling laws are also given. The results of the simulations are presented for several scenarios corresponding to different wakefield time/length scales and phase velocities. For the first time, FDI is considered for realistic conditions where the probe pulse dephases from the plasma wave and large frequency shifts are present. Limitations induced by these two effects are also discussed. A comparison with PAD is provided for the same set of parameters. Finally, in the last section, we state the conclusions.

\section{DIAGNOSTIC PRINCIPLES}

\section{A. Frequency-domain interferometry}

The purpose of FDI is the measurement of the phase shift experienced by a probe laser pulse traveling through an electron density plasma perturbation. The experimental principle is as follows: a high-intensity, ultrashort laser pulse (pump pulse) ionizes a gas at the focal region and excites an electron density perturbation (laser wakefield). A double pulse beam (probe and reference pulses) is focused on the same axis. Copropagating with the EPW [described by the electron density $n_{e}(z, t)$ ], the probe pulse 
will experience an optical phase shift $\Delta \phi$ proportional to $n_{e}(z, t)$, while the reference pulse, which precedes the pump pulse, propagates through the interaction region unperturbed. The relative phase shift between these two probing pulses is then measured by the FDI technique. The relative positions in time of the three laser pulses (reference, pump, and probe) are illustrated in Fig. 1.

The FDI technique is based on a temporal recombination of the probe and the reference pulses in a spectrometer. The temporal beating creates a system of fringes in the frequency domain. The position of the fringes depends on the relative phase between the probe pulse and the reference pulse. This phase difference $\Delta \phi$ can be obtained in a straightforward way from the power spectrum $I(\omega)$ recorded by a charge-coupled device (CCD) camera or diode-array detector. To extract the phase information from the spectral domain we calculate the inverse Fourier transform of $I(\omega)$

$$
\begin{aligned}
\mathcal{F} . \mathcal{T} .|I(\omega)|= & \frac{1}{\sqrt{2 \pi}} \int_{-\infty}^{\infty} I(\omega) \exp (i \omega t) d t \\
= & 2 h\left(t^{\prime}\right)+\exp (i \Delta \phi) h\left(t^{\prime}-\Delta t\right) \\
& -\exp (-i \Delta \phi) h\left(t^{\prime}+\Delta t\right),
\end{aligned}
$$

where $h\left(t^{\prime}\right)$ is the inverse Fourier transform of the original probe pulse. It is clear that the phase information is contained on the sidebands centered at $t^{\prime}= \pm \Delta t$. If $\Delta t$ is sufficiently large, the sidebands are separated from the autocorrelation term $h\left(t^{\prime}\right)$ at $t^{\prime}=0$, and it is possible to extract a complex value, which contains the phase information, by calculating the phase angle of $\mathcal{F} \cdot \mathcal{T} .|I(\omega)|$ at the $t^{\prime}= \pm \Delta t$ points.

It should be noted that in this diagnostic technique it is normally assumed that the group velocity of the probe beam and the phase velocity of the plasma wave are identical, i.e., $v_{g_{\text {probe }}}=v_{p}$. Therefore, it is assumed that the probe pulse always stays in phase with the density perturbation, sampling only a small portion of the plasma wakefield during propagation. Let us consider the "simplified" situation where the laser wakefield is given by

$$
n_{e}(z, t)=\delta n_{e}(z) \sin \left[k_{p}\left(z-v_{p} t\right)\right]+n_{e_{0}},
$$

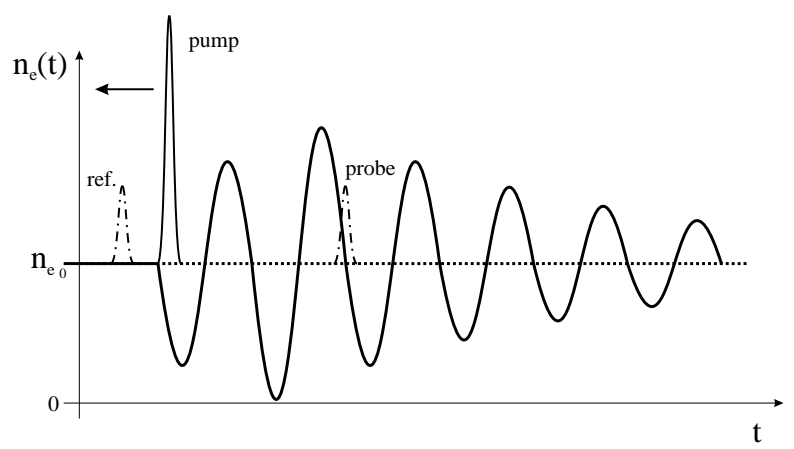

FIG. 1. Schematic visualization of the pump pulse, the trailing wakefield, and two probe pulses. where $v_{p}$ is the phase velocity and $k_{p}$ is the wave number of the density perturbation $\delta n_{e}$ excited in an homogeneous plasma background $n_{e_{0}}$. The maximum measured phase shift in the frequency-domain interferograms is the integrated phase shift of the probe pulse after propagating through the interaction region

$$
\Delta \phi=\int_{-\infty}^{\infty} \frac{2 \pi}{\lambda_{0}}\left[\eta_{\text {probe }}(z)-\eta_{\text {ref }}(z)\right] d z,
$$

where $\lambda_{0}$ is the wavelength of the probe and the reference pulses. The plasma refractive index seen by the probe pulse $\eta_{\text {probe }}(z)=\sqrt{1-\left[\delta n_{e}(z)+n_{e_{0}}\right] / n_{c}}$ and the plasma refractive index $\eta_{\text {ref }}(z)=\sqrt{1-n_{e_{0}} / n_{c}}$ seen by the reference laser pulse are determined by the amplitude of the laser wakefield $\delta n_{e}(z)$ and by the unmodulated plasma $n_{e_{0}}$, respectively (where $n_{c}$ is the critical density for the two laser pulses).

Finally, by sweeping the probe pulse along one or more periods of the plasma wave with a temporal delay line, it is possible to reconstruct the wakefield oscillation with an amplitude given by Eq. (3) and a wavelength identical to that of the plasma wave $\lambda_{p}=2 \pi / k_{p}$.

At this point it is important to notice that the above description $[3,4]$ of the phase shift experienced by the probe pulse leaves out the contribution of the frequency shift of the pulse. The dependence of the group velocity of the probe pulse on the local plasma density $v_{g_{\text {probe }}}\left(\delta n_{e}\right)$ is also not taken into account. In our treatment these two effects will be included.

Apart from these two approximations, one of the most important characteristics of this diagnostic technique is the high sensitivity to small density perturbations. This comes from the fact that in this interferometric technique the signal is placed on a carrier (frequencydomain fringes) and uses phase-sensitive lock-in detection in order to avoid stray light, pump leakage, and detector defect problems.

\section{B. Photon acceleration technique}

Photon acceleration was first proposed by Wilks et al. [11] to describe the frequency shift experienced by a probe laser pulse copropagating with a relativistic EPW. This designation has also been used to describe the frequency shift of electromagnetic (e.m.) waves in other configurations [12-14].

Let us consider an EPW described by $\delta n_{e}=$ $\delta n_{e_{0}} \sin \left(k_{p} \zeta\right)$, where $\zeta=z-v_{p} t$, and assume a low-intensity probe laser pulse centered around $\zeta=0$, with a pulse length $\sigma_{z} \ll 2 \pi / k_{p}$ propagating in the EPW. As we can see in Fig. 2, the local density at the front of the laser pulse will be smaller than that at the back of the pulse. Since the phase velocity is proportional to the plasma density, the phase velocity at the front of the pulse is slower than at its back: The phase peaks at the 


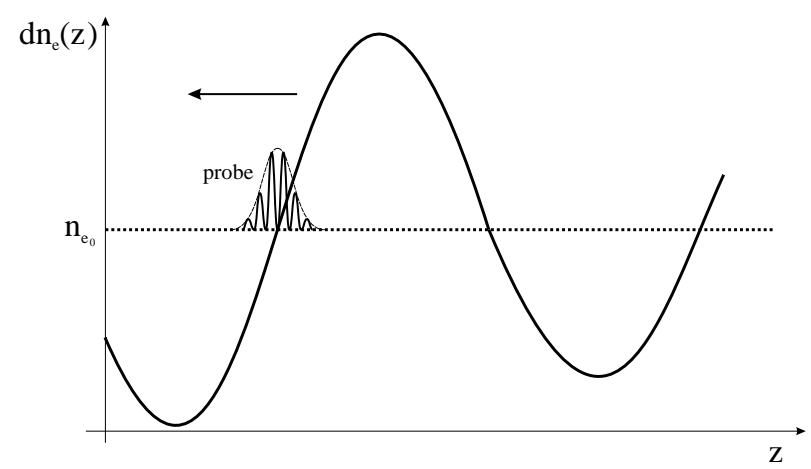

FIG. 2. Schematics of laser pulse frequency up-shifting by a plasma wave when $v_{g_{\text {probe }}} \simeq v_{p} \simeq c$.

back move faster than those at the front of the laser pulse (represented here as a wave packet), which means that the wavelength decreases and the frequency increases. For small frequency shifts, and considering that the laser pulse remains in phase with the plasma wave $v_{g_{\text {probe }}}=v_{p}$, the frequency shift is given by [15]

$$
\Delta \omega \simeq \frac{\omega_{p}^{2}}{2 \omega_{0}} \frac{\delta n_{e_{0}}}{n_{e_{0}}} \Delta z k_{p} \cos \left(k_{p} \zeta\right)
$$

where $\omega_{p}=\left(4 \pi n_{e 0} c^{2} / m_{e}\right)^{1 / 2}$ is the plasma frequency of the unperturbed plasma, $\omega_{0}$ is the frequency of the laser pulse, and $\Delta z$ is the propagation distance.

From Eq. (4) we can easily map the plasma wave by injecting the probe laser pulse at different positions of the plasma oscillation $\zeta$, as in the FDI diagnostic technique. Notice that Eq. (4) is valid only for very small frequency shifts $\Delta \omega \ll \omega_{0}$. This is usually true for present laser wakefield experiments, but in future experiments, with long propagation distances and large EPW amplitudes, the frequency shifts can be of the order of the frequency of the laser pulse. In this situation a more general theory must be used. Recently, the Hamiltonian formulation of photons $[14,16,17]$ was introduced as a new description of the frequency shift phenomena, providing a more powerful description of the photon acceleration mechanism. Generally, the solution of the ray-tracing equations can only be obtained numerically; however, fully analytical results can be achieved for some electron density perturbations, such as an ionization front. For instance, we can easily calculate the frequency shift which occurs when a wave packet (classical analog of a photon) crosses over an ionization front without reflection [14]

$$
\Delta \omega=\frac{\omega_{p 0}^{2}}{2 \omega_{0}} \frac{\beta}{1 \pm \beta},
$$

where the initial frequency of the photon is much higher than the maximum frequency of the plasma behind the ionization front, i.e., $\omega_{0} \gg \omega_{p 0}$. The sign $+(-)$ refers to counterpropagation (copropagation) where $v_{p}=\beta c$ is the velocity of the ionization front.
Recent experimental results [8] have shown a very good agreement with this ray-tracing formalism. The results of the frequency up-shift in the copropagation and counterpropagation setups of this experiment allow us to determine the electron plasma density and the ionization front velocity by using the 2D version of Eq. (5) [8]. This clearly points to the feasibility of PAD for relativistic coherent structures in laser produced plasmas. It is very important to mention that in this new description of the frequency-shift diagnostic technique, the assumption $v_{g_{\text {probe }}}=v_{p}$ is no longer necessary, and the limitation to small frequency shifts does not exist.

\section{RAY-TRACING SIMULATIONS}

The results presented in this Section are based on the numerical integration of the photon (short laser pulse) trajectories described by the ray-tracing equations, in the presence of a laser wakefield. The probe pulse length is not considered, and the wave packet is characterized only by its central frequency and central wave number. When the laser pulse propagates in the presence of an electron density perturbation, the linear dispersion relation

$$
\omega^{2}-k^{2} c^{2}-\omega_{p}^{2}\left(z-v_{p} t\right)=0
$$

is assumed valid, where $\omega$ is the frequency, $k$ is the wave number, and $\omega_{p}\left(z-v_{p} t\right)$ is the local electron plasma frequency associated with the electron density perturbation propagating with phase velocity $v_{p}$. Equation (6) is valid in two conditions: (i) The electromagnetic wave packet does not disturb the electron density perturbation and (ii) the time scale $\tau_{p}$ (length scale) of the perturbation is much longer than the period (wavelength) of the wave packet, i.e., $2 \pi / \omega \ll \tau_{p}\left(2 \pi / k \ll \tau_{p} v_{p}\right)$. These conditions are usually satisfied when probing is performed by a low-intensity, ultrashort pulse, with a central frequency much higher than the electron plasma frequency. The dispersion relation (6) can be inverted to express the frequency $\omega$ as a function of the other variables $k$ and $t$. Assuming 1D propagation along the $z$ direction, we can then obtain the ray-tracing equations

$$
\begin{gathered}
\frac{d z}{d t}=\frac{\partial \omega}{\partial k}=c \sqrt{1-\frac{\omega_{p}^{2}(z, t)}{\omega^{2}}}, \\
\frac{d k}{d t}=-\frac{\partial \omega}{\partial z}=-\frac{1}{2 \omega} \frac{\partial \omega_{p}^{2}(z, t)}{\partial z} .
\end{gathered}
$$

These equations allow us to calculate the wave number of the probe laser pulse at any point of its trajectory. Knowing the wave number we then obtain, in a straightforward way, the frequency shift by using the dispersion relation in Eq. (6)

$$
\Delta \omega(z, t)=\sqrt{k^{2}(z, t) c^{2}+\omega_{p}^{2}(z, t)}-\omega_{0}
$$


where $k(z, t)$ is the pulse wave number and $\omega_{p}(z, t)$ is the plasma frequency of the wakefield at a point $(z, t)$ along the laser pulse trajectory. $\omega_{0}$ is the pulse frequency before interacting with the plasma wave. The phase shift $\phi$ experienced by the laser pulse in the wakefield is determined by using the same ray-tracing trajectories and writing

$$
\phi_{\text {probe }}(z, t)=\int_{0}^{z} k(z, t) d z-\int_{0}^{t} \omega(z, t) d t,
$$

where $k(z, t)$ and $\omega(z, t)$ are the wave number and frequency along the ray-tracing trajectory. We can then determine the total phase shift relative to a pulse propagating in an unmodulated plasma

$$
\Delta \phi(z, t)=\phi_{\text {probe }}(z, t)-\phi_{\text {ref }}(z, t) .
$$

The phase of the reference pulse can be written in the form

$$
\phi_{\mathrm{ref}}(z, t)=\int_{0}^{z} k_{0}-\frac{\omega_{0}}{v_{g_{\mathrm{ref}}}} d z
$$

where $k_{0}$ and $\omega_{0}$ are the wave number and the frequency of the reference pulse. The group velocity of the reference laser pulse $v_{g_{\text {ref }}}$ is given by

$$
v_{g_{\text {ref }}}=\frac{k_{0} c^{2}}{\sqrt{k_{0}^{2} c^{2}+\omega_{p_{0}}^{2}}}=c \sqrt{1-\frac{\omega_{p_{0}}^{2}}{\omega_{0}^{2}}},
$$

where $\omega_{p_{0}}$ is the plasma frequency of the homogenous unmodulated plasma.

\section{A. Laser wakefield scaling laws}

For the sake of completeness, we present here the expressions for the laser wakefield excitation in the linear nonrelativistic two-dimensional (2D) regime. Using the solution of the linearized 2D fluid equations given by $[18,19]$, we have, in terms of the normalized vector potential $a_{L}$ of the pump pulse, the second-order density perturbation

$$
\begin{aligned}
\frac{\delta n_{e}}{n_{e_{0}}}= & \frac{a_{L}^{2}(\zeta, r)}{2}+k_{p} \int_{\zeta}^{\infty} \sin \left[k_{p}\left(\zeta-\zeta^{\prime}\right)\right] \frac{a_{L}^{2}(\zeta, r)}{2} d \zeta^{\prime} \\
& -\frac{1}{k_{p}} \int_{\zeta}^{\infty} \sin \left[k_{p}\left(\zeta-\zeta^{\prime}\right)\right] \\
& \times\left[\frac{1}{r} \frac{\partial}{\partial r} r \frac{\partial}{\partial r} \frac{a_{L}^{2}(\zeta, r)}{2}\right] d \zeta^{\prime},
\end{aligned}
$$

where $r$ is the radial coordinate describing the distance from the pump laser propagation axis. Assuming that the pump beam is Gaussian in the radial direction and that the longitudinal pulse shape is also Gaussian, we have

$$
a_{L}^{2}(\zeta, r)=a_{0}^{2} \exp \left[-\left(\zeta / \sigma_{z}\right)^{2}\right] \exp \left[-\left(r / \sigma_{r}\right)^{2}\right] .
$$

Inserting the above identity in (14), we can obtain [20]

$$
\begin{aligned}
\frac{\delta n_{e}}{n_{e_{0}}}= & \frac{a_{0}^{2}}{2} \exp \left[-\left(r / \sigma_{r}\right)^{2}\right]\left\{1+\frac{4}{\left(k_{p} \sigma_{r}\right)^{2}}\left[1-\left(\frac{r}{\sigma_{r}}\right)^{2}\right]\right\} \\
& \times \sqrt{\pi}\left\{k_{p} \sigma_{z} \exp \left[-\left(k_{p} \sigma_{z}\right)^{2} / 4\right]\right\} \sin \left(k_{p} \zeta\right),
\end{aligned}
$$

where $k_{p}=\left(4 \pi e^{2} n_{e} / m_{e} c^{2}\right)^{1 / 2}$ is the wave number of the plasma wave.

For a better understanding of our calculations, we will work with laboratory-oriented parameters: pump pulse energy $E_{0}$, pulse width $\sigma_{z}=c \tau /(2 \sqrt{\ln 2})$ (where $\tau$ is the FWHM pulse width), and central wavelength $\lambda_{0}$. In terms of the new parameters and using the following relations

$$
\begin{gathered}
a_{0}^{2}=\frac{I}{\left(\frac{\pi^{2}}{2} \frac{m_{e} c^{2}}{r_{e} / c} \frac{1}{\pi \lambda_{0}^{2}}\right)}, \\
I=\frac{c}{\sqrt{\pi}} \frac{E_{0}}{\pi \sigma_{z} \sigma_{r}^{2}},
\end{gathered}
$$

where $r_{e}$ is the classical electron radius and $m_{e}$ is its mass, we rewrite (16) as

$$
\begin{aligned}
\frac{\delta n_{e}}{n_{e_{0}}}= & \frac{2}{\pi^{2} \sqrt{\pi}}\left(\frac{\lambda r_{e}}{\sigma_{r}^{2}}\right)^{2} \frac{1}{\sqrt{r_{e}^{3} n_{e}}} \frac{E_{0}}{m_{e} c^{2}} \\
& \times \exp \left[-\left(r / \sigma_{r}\right)^{2}\right] \exp \left(-\pi r_{e} n_{e} \sigma_{z}^{2}\right) \\
& \times\left\{\pi r_{e} n_{e} \sigma_{r}^{2}+\left[1-\left(\frac{r}{\sigma_{r}}\right)^{2}\right]\right\} \sin \left(k_{p} \zeta\right) .
\end{aligned}
$$

For a Gaussian focus, $\sigma_{r}$ varies longitudinally as $\sigma_{r}^{2}=$ $\sigma_{r_{0}}^{2}\left[1+\left(z / z_{r}\right)^{2}\right]$, where the Rayleigh length is $z_{r}=$ $(2 \pi / \lambda) \sigma_{r_{0}}^{2}$. Of particular interest is the electron density $n_{e_{0}}$ which maximizes $\delta n_{e}$. For this we use the reduced expression, in the $2 \mathrm{D}$ limit, for the resonant density $n_{\text {res }}=$ $1 / 2 \pi r_{e} \sigma_{z}^{2}[20]$.

Finally, the requirement that $\delta n_{e} / n_{e_{0}} \leq 1$ in the laser focus imposes a minimum size to the laser focal spot $\sigma_{r_{0}}$. We used this limit in our simulations in order to optimize our density perturbation. For simplicity, we have decided to analyze, in all the simulations, only the trajectories at $r=0$ (1D simulations), where Eq. (19) reduces to

$$
\begin{aligned}
\frac{\delta n_{e}}{n_{e_{0}}}= & \frac{2}{\pi^{2} \sqrt{\pi}}\left(\frac{\lambda r_{e}}{\sigma_{r}^{2}}\right)^{2} \frac{1}{\sqrt{r_{e}^{3} n_{e}}} \frac{E_{0}}{m_{e} c^{2}} \exp \left(-\pi r_{e} n_{e} \sigma_{z}^{2}\right) \\
& \times\left(\pi r_{e} n_{e} \sigma_{r}^{2}+1\right) \sin \left(k_{p} \zeta\right) .
\end{aligned}
$$

\section{B. Propagation velocity effects}

As mentioned in the previous sections, it is currently assumed in the FDI diagnostic $[3,4]$ that the probe laser pulse always stays in phase with the plasma wave, i.e., $v_{g_{\text {probe }}}=v_{p}$. This is not valid for two reasons: (i) The probe group velocity depends on the local electron plasma density $v_{g_{\text {probe }}}(z, t)=c \sqrt{1-\omega_{p}^{2}(z, t) / \omega}$ at each point of the pulse trajectory [see Eqs. (7) and (8)], and (ii) the phase velocity of the wakefield $v_{p}$, which is nearly equal to the group velocity of the pump laser pulse, can be considerably different from the velocity given by the linear dispersion relation in a plasma $\left(v_{g_{\text {pump }}} \neq\right.$ $\left.c \sqrt{1-\omega_{p}^{2} / \omega}\right)$, due to nonlinear and 3D effects. These two aspects of the velocity effects in the probe pulse are discussed separately in this subsection. 
In our simulations we have considered typical parameters for the pump laser pulse used in recent laser wakefield experiments [3,4]: $E_{0}=2.5 \mathrm{~mJ}, \tau_{\text {pump }}=100 \mathrm{fs}\left(\sigma_{z}=\right.$ $18 \mu \mathrm{m})$ which can be focused down to $\sigma_{r_{0}} \approx 3.6 \mu \mathrm{m}$ (for $\delta n_{e} / n_{e_{0}} \leq 1$ ) in the $2 \mathrm{D}$ resonant plasma density of $n_{e_{0}} \approx 1.74 \times 10^{17} \mathrm{~cm}^{-3}$. Since in this type of experiments the probe pulse is usually a small fraction of the pump beam, we use the same wavelength $\lambda_{0}=800 \mathrm{~nm}$ for both beams.

In Figs. 3(a) and 3(b) we present the phase and frequency shift of the probe pulse, after interacting with the plasma perturbation, for different time and space delays. These delays enable us to insert the probe pulse at different positions of the laser wakefield and to sample different regions of the plasma wave oscillations. In Fig. 3(a) we compare the phase shift obtained for $v_{g_{\text {probe }}}=$ $v_{p}$ (dashed curve) with the phase shift, considering the probe pulse group velocity as a function of the local density $v_{g_{\text {probe }}}\left[\delta n_{e}(z, t)\right]$ (solid curve). The phase velocity of the plasma perturbation is given, in this case, by the group velocity of a pump laser pulse in a homogeneous unmodulated plasma density $n_{e_{0}}\left(v_{p}=c \sqrt{1-\omega_{p_{0}}^{2} / \omega^{2}}\right)$. From Fig. 3 it is clear that the obtained phase shift

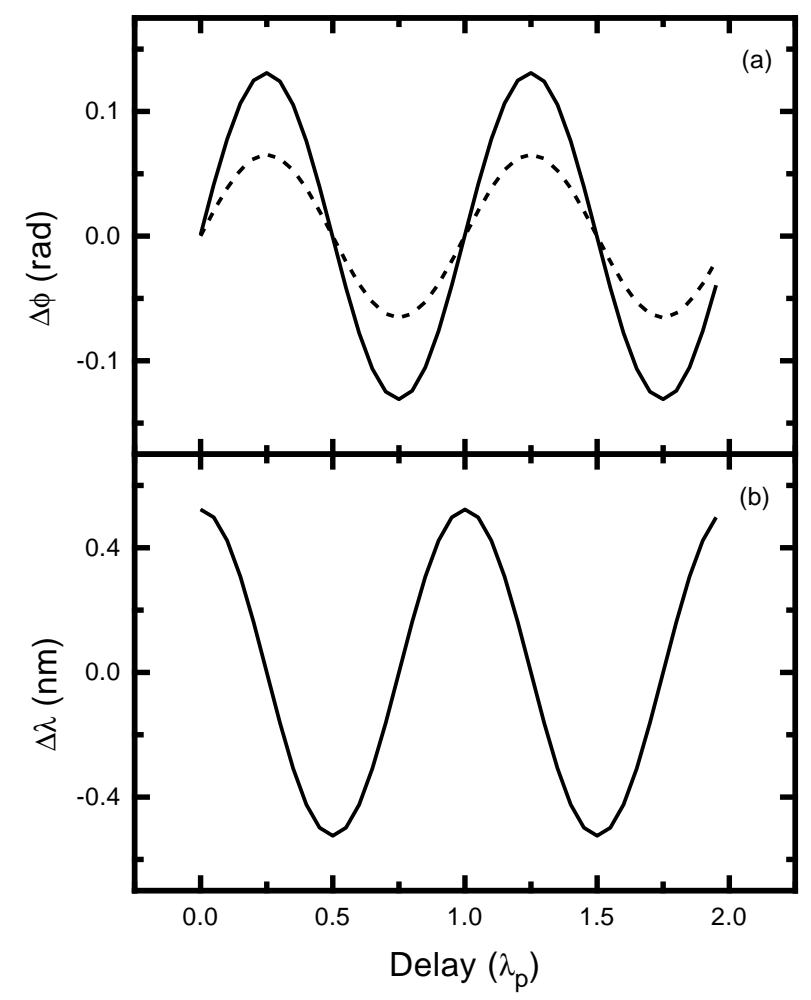

FIG. 3. Wakefield oscillation map given by the numerical results for (a) phase shift and (b) frequency shift for different delays, assuming that $v_{g_{\text {probe }}}=v_{p}$ (dashed curves) or that the probe pulse group velocity depends on the local plasma density $v_{g_{\text {probe }}}\left[\delta n_{e}(z, t)\right]$ (solid curves). In (b) the dashed curve and the solid curve overlap. The pump laser pulse parameters are $E_{0}=2.5 \mathrm{~mJ}, \tau_{\text {pump }}=100 \mathrm{fs}$. oscillation is amplified when we consider that the probe group velocity depends on the local electron plasma density, so the measured phase shift can mislead us in the evaluation of the plasma wave amplitude. This can be explained by the fact that we are comparing the probe pulse phase and the reference pulse phase at the same spatial point but at different times, due to the difference in their group velocities. Comparatively, the frequency shift [see Fig. 3(b)] remains the same in both cases.

Now let us assume that the phase velocity $v_{p}$ of the wakefield is no longer equal to the group velocity of the pump, but it is an independent parameter. For $v_{p}=0.9 c$ the simulations are shown (solid curves) in Figs. 4(a) and 4(b) for the probe pulse phase and frequency shifts, respectively. Comparing these results with the previous ones (dashed curves), we notice that the wavelength of the phase shift and the frequency shift oscillations are enlarged by nearly $12 \%$ and that the amplitudes are slightly smaller. So, in this case, the wavelength measured by the probe pulse phase or by the frequency shift will be larger than the wavelength of plasma wakefield ( $\lambda_{\text {measured }} \approx 1.12 \lambda_{p}$ for $v_{p}=0.9 c$ ). To overcome this problem we need an independent measure of the phase velocity of the plasma wakefield, which can be obtained by comparing the frequency shift in copropagation and in counterpropagation, as demonstrated in recent photon acceleration experiments [8].

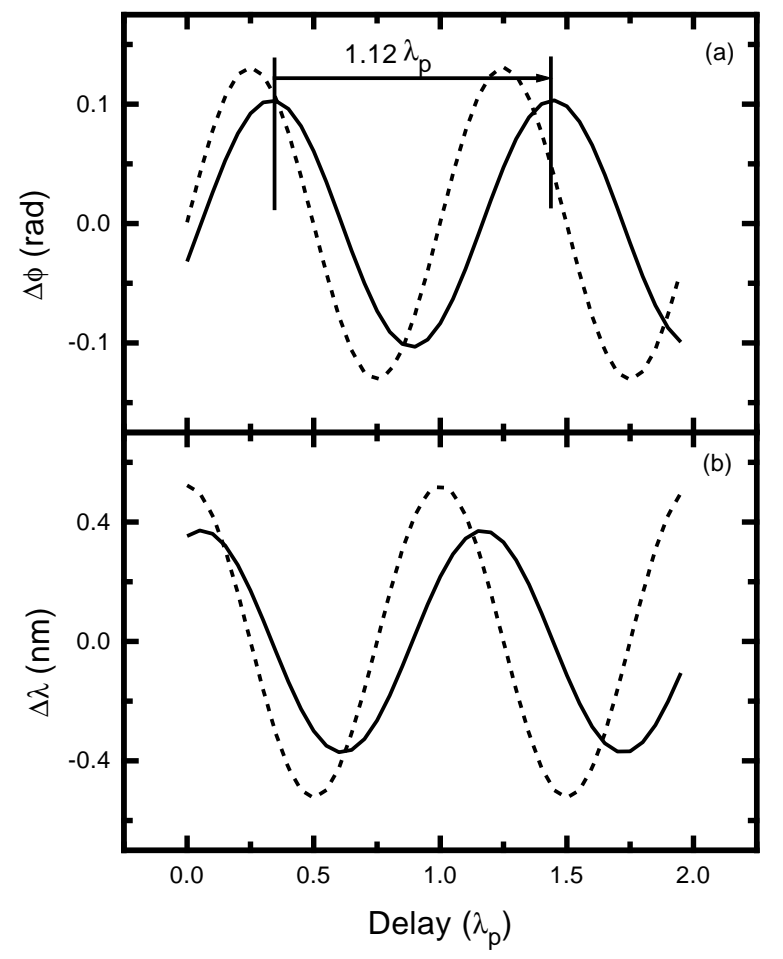

FIG. 4. Wakefield oscillation map given by (a) phase shift and (b) frequency shift, assuming that the propagation velocity of the plasma wave $v_{p}=v_{g_{\text {pump }}}\left(n_{e_{0}}\right)$ (dashed curves) or that $v_{p}=0.9 c$ (solid curves). $E_{0} \stackrel{g \text { ump }}{=} 2.5 \mathrm{~mJ}, \tau_{\text {pump }}=100 \mathrm{fs}$. 


\section{Large frequency shifts effects}

Another assumption, normally taken in the measurements of the laser wakefield properties, is that the frequency shift experienced by the probe pulse is neglegible and that it can be neglected for phase shift calculations. This is usually true in present day experiments, but in the near future it will be possible to excite larger EPW with the help of more powerful lasers and to make them propagate along longer distances [21]. This will lead to much larger frequency shifts of the probe laser pulse. In order to examine the importance of a large frequency shift in FDI we have changed the pump laser pulse parameters: We have increased the pulse energy to $E_{0}=100 \mathrm{~mJ}$ and compressed the pulse duration to $\tau_{\text {pump }}=30 \mathrm{fs}\left(\sigma_{z} \approx 5.4 \mu \mathrm{m}\right)$. The new plasma wakefield perturbation obtained from the scaling laws of Sect. IIIA is $\delta n_{e} \approx 1.93 \times 10^{18} \mathrm{~cm}^{-3}$ in the laser focus $\left(\sigma_{r_{0}} \approx 8.2 \mu \mathrm{m}\right.$ for $\left.\delta n_{e} / n_{e_{0}} \approx 1\right)$.

From Fig. 5(b) we can see that the frequency shift experienced by the probe pulse is of the same order of its initial frequency. In this simulation, the frequency shift can go up to $\Delta \lambda=\left|\lambda_{0}-\lambda\right| \approx 200 \mathrm{~nm}$ for a probe laser wavelength of $\lambda_{0}=800 \mathrm{~nm}$. Like in the simulations of Fig. 3, the frequency shift reaches its positive and negative maximum values when the pulse is positioned in the regions of the strongest negative and

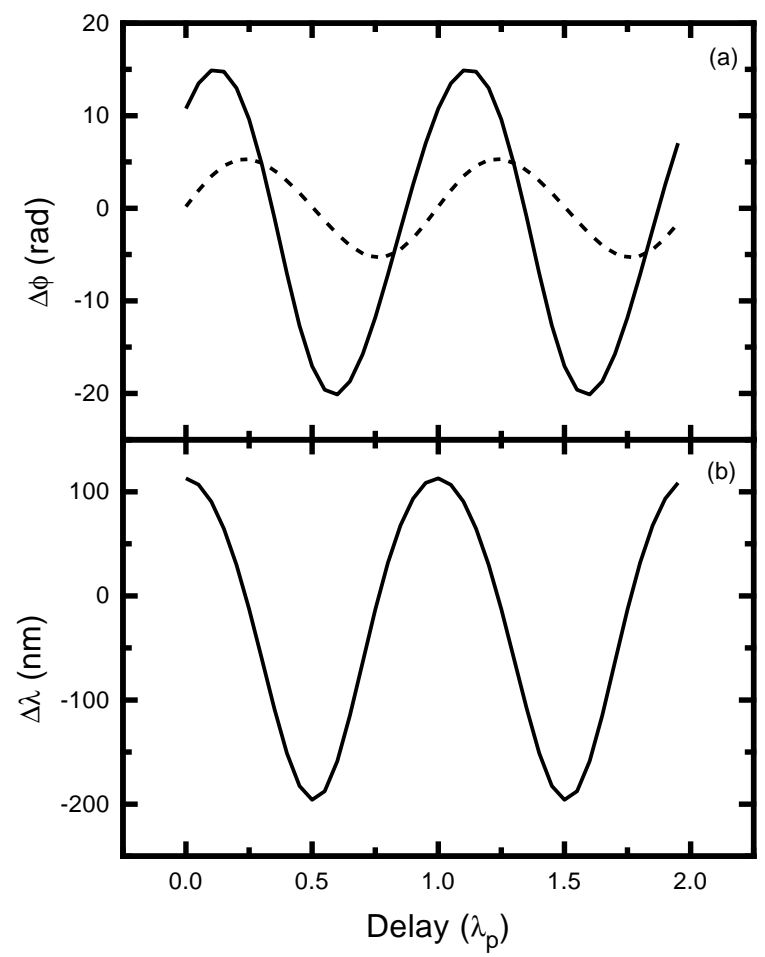

FIG. 5. Wakefield oscillation map giving (a) the phase shift and (b) the frequency shift for the laser pulse parameters: $E_{0}=100 \mathrm{~mJ}, \tau_{\text {pump }}=30 \mathrm{fs}$. The calculated phase shifts in (a) are obtained by neglecting the frequency shift of the probe pulse (dashed curve) or by retaining it (solid curve). positive gradients of the electron density perturbation, respectively. But in the present situation the maximum frequency up-shift $\left(\Delta \lambda_{+} \simeq 113 \mathrm{~nm}\right)$ is much smaller than the maximum frequency down-shift $\left(\Delta \lambda_{-} \simeq 196 \mathrm{~nm}\right)$. This effect arises from the fact that the frequency shift at each point of the ray-tracing trajectory of the probe pulse is inversely proportional to its frequency [see Eq. (8)].

In Fig. 5(a) we present the phase shifts of the probe pulse given by Eqs. (10)-(12) including the frequency shift contribution (solid curve) or neglecting those contributions (dashed curve). Comparing these two curves we can notice that not only the phase shift oscillations become much stronger, but a similar nonlinear behavior is also present, i.e., $\left|\Delta \phi_{-}\right|>\left|\Delta \phi_{+}\right|$. Figure 6 provides the explanation for this effect. This is a typical plot of the frequency shift $\Delta \lambda$ (solid) and phase shift $\Delta \phi$ (dashed) along the probe pulse trajectory through the laser wakefield. It is obvious that the phase shift of the probe pulse does not tend to a constant value, in contrast with the frequency shift. This is due to the fact that the final frequency of the probe pulse is very different from the reference pulse frequency (which is constant), and the phase difference between these two pulses increases continuously as long as they propagate inside the plasma. Therefore, for a large frequency shift, the measured phase shift will be strongly dependent on the propagation length.

This can lead to phase shifts several times larger than $\pi$. For instance, in the simulations of Fig. 5, where the total propagation distance inside the plasma is $\Delta z \approx 30 z_{r}$ [22], the phase shift oscillations reach amplitudes of $\approx$ $11 \pi$ (peak to peak). This is an additional difficulty for the FDI technique. In fact, the time-delay step $\Delta t$ for this diagnostic technique must be small enough to enable us to count all of the displaced fringes in the frequency-domain interferograms. The upper limit for this time-delay step $\Delta t$ is given by

$$
\Delta t \leq \frac{T_{p}}{4\left(\Delta \phi_{p p} / \pi\right)}
$$

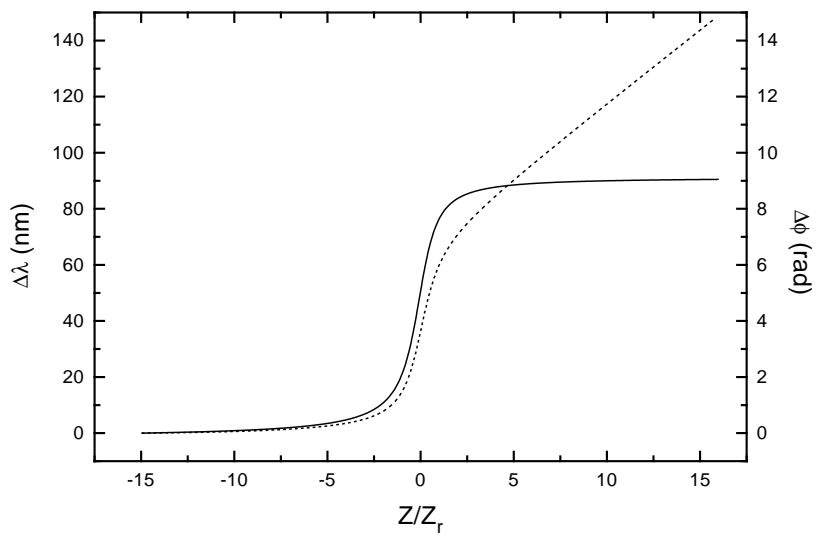

FIG. 6. Typical plot of the frequency shift $\Delta \lambda$ (solid) and phase shift $\Delta \phi$ (dashed) at each point of the probe pulse trajectory centered at $z / z_{r}=0$. 


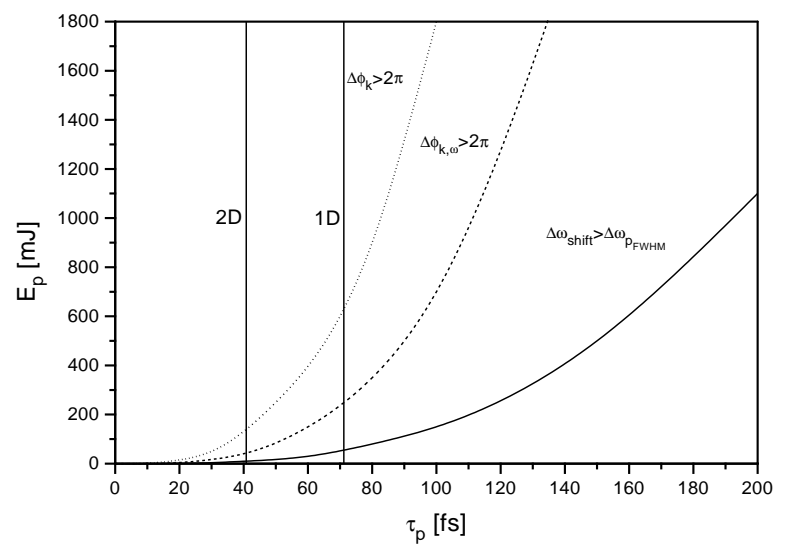

FIG. 7. Map of the pump laser pulse parameters (pulse energy versus length). Solid curve defines the limit where the frequency shift is equal to the spectral width of the probe pulse $\left(\Delta \omega_{\text {shift }}=\Delta \omega_{p_{\text {FWHM }}}\right)$. Dashed and dotted curves define the limits of applicability of the FDI. The vertical lines indicate the $100 \mathrm{GeV} / m$ goal for the future plasma accelerators as predicted by the $2 \mathrm{D}$ and $1 \mathrm{D}$ scaling laws.

where $T_{p}=\lambda_{p} / v_{p}$ is the plasma wave period and $\Delta \phi_{p p}$ is the expected phase shift oscillation amplitude (peak to peak).

We will now discuss the limits of application for each of the two diagnostic techniques. In order to illustrate these limits we have built up a map representing the pump laser parameters, energy $E_{p}$ versus pulse length $\tau_{p}$ (see Fig. 7). For each set of parameters, the corresponding laser wakefield scaling is obtained for the optimized situation $\delta n_{e} / n_{e_{0}} \approx 1$ for the resonant density in the 2D limit. The criteria used to define the limiting curves are as follows. The solid curve is given by the condition of frequency shift $\Delta \omega_{\text {shift }}$ measured by the PAD technique equal to the spectral width of the probe pulse $\Delta \omega_{p_{\text {гшнм }}}$

$$
\Delta \omega_{\text {shift }}=\Delta \omega_{p_{\text {FWHM }}} .
$$

Since, from an experimental point of view, it is very difficult to measure a frequency shift smaller than the spectral width, this technique is only valid above this curve.

The FDI technique is limited by the measured phase shift $\Delta \phi$, which must be lower than $2 \pi$. We have plotted two curves above which the FDI technique is not valid: one (dotted curve) considering the phase shift as given by the refraction index $\Delta \phi_{k}$, Eq. (3), and the other (dashed curve) retaining the contribution of the frequency variation $\Delta \phi_{k, \omega}$, Eqs. (10)-(12). In this map, we also represent two lines defining the $100 \mathrm{GeV} / \mathrm{m}$ goal for accelerating gradients, already measured by indirect techniques in recent experiments [23]. The lines correspond to the 2D and 1D limits of the resonant density. A close analysis of this map clearly shows that photon acceleration is the most appropriate diagnostic technique for the future laser wakefield accelerators.

\section{CONCLUSIONS}

In this work we have presented a detailed comparison between two different diagnostic techniques for laser wakefields. Using the ray-tracing equations we considered, for the first time, the group velocity dispersion and frequency-shift effects, which have been neglected until now in the current literature on FDI, but can be of great importance in the interpretation of future experiments, as we have shown in this paper. By simulating the timedelay scanning of the plasma oscillations we were able to identify and clarify the impact of these effects in both diagnostic techniques.

Our numerical simulations show that for FDI the wakefield oscillation reconstructed from the measured phase shifts is significantly modified in both amplitude and frequency, if the dispersion effects in the probe beam are included and/or assuming that the plasma wave phase velocity is different from the group velocity of the pump laser beam. On the contrary, for the PAD technique, the results are not affected by these dispersion effects, which, in fact, are fundamental processes in the PAD.

We have also considered experimental parameters relevant to future laser wakefield experiments. In these conditions, the frequency shift of the probe beam cannot be neglected. Moreover, we have studied the influence of a large frequency shift on the phase measurements made with the FDI. In this case the frequency of the probe pulse is completely different from that of the reference pulse. Thus the phase difference will depend not only on the plasma length but also on the dispersive optics installed in their optical path before reaching the detector device. This fact, added to the complexity of measuring phase shifts much larger than $\pi$ and the difficulty of using the FDI of two laser beams with very different frequencies, will be the major drawback of this laser wakefield diagnostic technique. Comparatively, the large frequency shifts play in favor of the PAD technique due to the fact that the extraneous data contributions, such as stray light, pump leakage, and detector defects, are no longer a technical problem. For all of the reasons discussed above, PAD is the most promising diagnostic for large amplitude plasma waves in future laser wakefield accelerator experiments.

\section{ACKNOWLEDGMENTS}

We thank G. Figueira for his comments on this paper. The work of J.M.D. was supported by a grant from JNICT-PRAXIS XXI.

[1] T. Tajima and J. M. Dawson, Phys. Rev. Lett. 43, 267 (1979).

[2] J. B. Rosenzweig et al., Phys. Rev. Lett. 61, 98 (1988).

[3] J. R. Marquès et al., Phys. Rev. Lett. 76, 3566 (1996). 
[4] C. W. Siders et al., Phys. Rev. Lett. 76, 3570 (1996).

[5] E. Tokunaga, A. Terasaki, and T. Kobayashi, Opt. Lett. 17, 1131 (1992).

[6] J.P. Geindre et al., Opt. Lett. 19, 1997 (1994).

[7] W. M. Wood, C. W. Siders, and M.C. Downer, IEEE Trans. Plasma Sci. 21, 20 (1993).

[8] J. M. Dias et al., Phys. Rev. Lett. 78, 4773 (1997).

[9] C. D. Decker and W. B. Mori, Phys. Rev. Lett. 72, 490 (1994).

[10] E. Esarey, P. Sprangle, M. Pilloff, and J. Krall, J. Opt. Soc. Am. B 12, 1695 (1995).

[11] S. C. Wilks et al., Phys. Rev. Lett. 62, 2600 (1989).

[12] M. Lampe, E. Ott, and J.H. Walker, Phys. Fluids 10, 42 (1978).

[13] J. T. Mendonça, J. Plasma Phys. 22, 15 (1979).

[14] J. T. Mendonça and L. O. e Silva, Phys. Rev. E 49, 3520 (1994).
[15] E. Esarey, A. Ting, and P. Sprangle, Phys. Rev. A 42, 3526 (1990).

[16] L. O. e Silva and J. T. Mendonça, IEEE Trans. Plasma Sci. 24, 316 (1996).

[17] L. O. e Silva and J. T. Mendonça, Phys. Rev. E 57, 3423 (1998).

[18] L. M. Gorbunov and V. I. Kirsanov, Sov. Phys. JETP 66, 290 (1987).

[19] E. Esarey, A. Ting, P. Sprangle, and G. Joyce, Comments Plasma Phys. Control. Fusion 12, 192 (1989).

[20] C. W. Siders et al., IEEE Trans. Plasma Sci. 24, 301 (1996).

[21] P. Sprangle et al., Phys. Rev. Lett. 69, 2200 (1992).

[22] C. G. Durfee III and H. M. Milchberg, Phys. Rev. Lett. 71, 2409 (1993).

[23] A. Modena et al., Nature (London) 377, 606 (1995). 